\title{
3D Body Scanning Measurement System Associated with RF Imaging, Zero-padding and Parallel Processing
}

\author{
HyungTae Kim ${ }^{1}$, KyungChan $\mathrm{Jin}^{1}$, SeungTaek Kim${ }^{1}$, Jongseok Kim ${ }^{1}$, and Seung-Bok Choi ${ }^{2}$ \\ ${ }^{1}$ Smart Manufacturing Technology Group, KITECH, YangDae-GiRo road, 89, 331-825, CheonAn, South Korea \\ 2 Smart Structures and Systems Laboratory, Department of Mechanical Engineering, Inha University, Incheon 402-751, \\ South Korea, seungbok@inha.ac.kr
}

\begin{abstract}
This work presents a novel signal processing method for high-speed 3D body measurements using millimeter waves with a general processing unit (GPU) and zero-padding fast Fourier transform (ZPFFT). The proposed measurement system consists of a radio-frequency (RF) antenna array for a penetrable measurement, a high-speed analog-to-digital converter (ADC) for significant data acquisition, and a general processing unit for fast signal processing. The RF waves of the transmitter and the receiver are converted to real and imaginary signals that are sampled by a high-speed ADC and synchronized with the kinematic positions of the scanner. Because the distance between the surface and the antenna is related to the peak frequency of the conjugate signals, a fast Fourier transform (FFT) is applied to the signal processing after the sampling. The sampling time is finite owing to a short scanning time, and the physical resolution needs to be increased; further, zero-padding is applied to interpolate the spectra of the sampled signals to consider a $1 / \mathrm{m}$ floating point frequency. The GPU and parallel algorithm are applied to accelerate the speed of the ZPFFT because of the large number of additional mathematical operations of the ZPFFT. 3D body images are finally obtained by spectrograms that are the arrangement of the ZPFFT in a 3D space.
\end{abstract}

Keywords: Whole body scanner, RF imaging, general processing unit, parallel processing, zero-padding.

\section{INTRODUCTION}

Whole body scanners are used for security surveillance and they detect hidden hazards in a human body using RF waves. The scanner usually presents $3 \mathrm{D}$ images, hence, high-speed sampling and signal processing for the reconstruction are critical in commercial systems. The RF band used for this purpose is usually called a millimeter wave, whose wavelength is approximately between submillimeters and tens of millimeters. The millimeter wave can be propagated in air, and can penetrate dielectric materials but it is reflected from conductive materials [1], [2]. According to the study by Bjarnason et al., the transmissibility loss of RF waves under $200 \mathrm{GHz}$ is not more than $2 \mathrm{~dB}$ for most clothing and fabric materials such as rayon, nylon, silk, and wool as well as for leather [3].

The transmissibility of RF waves is applied in security systems for detecting weapons and constructing profiles of objects [4]. Dallinger et al. developed a weapon detection system using an RF lens antenna and they tested the system to detect hidden weapons in the upper body [5]. Martin constructed a passive RF imaging system to detect a metal gun placed among clothes. The system acquired RF images at frame rates of $30 \mathrm{~Hz}$ and $128 \times 192$ pixels [6]. The Pacific northwest national laboratory (PNNL), a leader in RF imaging, reported 2D photographic imaging as well as 3D cylindrical imaging systems. Sheen et al. proposed an RF holographic imaging system for concealed weapon detection in high security check points [4]. They developed a 3D imaging system to measure the depth of dielectric objects on the human body. The depth is detected at the peaks in the cylindrical spectrum images [7]. They also reported a highresolution imaging system and applied the holographic technique to detect a gun under leather [8]. Rappaport led a research project for whole body imaging using various sources. The system consisted of a transmitter, a reflector, and a mechanical stage. A virtual body was constructed after measuring the data and calculating the synthetic aperture radar (SAR) images [9]. Tan et al. proposed an RF imaging algorithm for a spherical-wave with a continuous frequency modulation. An array of antennas is arranged in a vertical direction and they surround the human body in cylindrical coordinates. A reconstruction procedure composed of several steps of inverse fast Fourier transform (IFFT) and FFT [10] was demonstrated. RF wave imaging has potential applications in the fashion and apparel industries because a person does not have to take off his/her clothing, and physical contact is not required to measure the body shape [11]. PNNL developed a 3D holographic scanning system 
that can measure body indices such as the circumference of the head, neck, chest, waist, and hip. The system developed by PNNL rotates an antenna array around a person and collects the reflective responses of the RF wave. The 3D data is a type of point-cloud and is presented using an avatar [12], [13].

These studies focus on surface measuring methods using RF waves and they do not include efficient signal processing methods after the measurements. The millimeter wave is disadvantageous for body measurements because the distance accuracy is less due to the lack of physical resolution. However, it has been the only available medium that penetrates for fabric materials without causing bionic damage to date. Current studies on millimeter imaging focus on increasing the RF frequency; however, the physical resolution remains lower than the conventional imaging. Another method of increasing the distance accuracy is to extend the sampling length for the FFT and by considering a $1 / \mathrm{m}$ floating frequency for the spectrum. However, the sampling length is limited owing to a finite measuring time; therefore, spectrum interpolation and floating point frequency can be applied to estimate the distance accurately [14]. Zero-padding shows an interpolation effect on the spectrum, that can detect a body surface more accurately and smoothen an image. However, the processing time will increase for obtaining a 3D image because of the large number of sampled signals and enormous computations of the ZPFFT. A GPU with parallel algorithms can be utilized to reduce the processing time because the GPU simultaneously calculates the same patterns as the mathematical operations. Consequently, the ZPFFT and the GPU are necessary to detect the body surface accurately in a short time.

In this study, the ZPFFT is applied to consider a $1 / \mathrm{m}$ floating point frequency and interpolate the spectra because of a finite time sampling. The amount of acquired data and the operations of the ZPFFT are significant; the use of a GPU for the ZPFFT is necessary for reducing the processing time. Experiments with the proposed method were carried out using a wave body scanner and a dummy body.

\section{BASIC PRINCIPLES}

\section{A. Concept of a scanning system}

The scanning system proposed in this work consists of an RF antenna array, a rotation mechanism, controllers, a server including a GPU and a high-speed data acquisition (DAQ) board, as shown in Fig.1. [15]. The rotation unit is installed on top of a frame and the antenna array is attached to the end of a horizontal bar that is connected to the rotation unit. The antenna array is vertically established facing the center of the rotation. The target object is a dummy man covered with copper foil. It is placed in the center and the rotation of the antenna array is done as per the cylindrical coordinates, as proposed by Sheen et al. [4]. The distance between the target object and the antenna array is represented by the radial coordinate, $r$, the rotation angle is $\theta$, and the vertical position of the antenna in the array is $\mathrm{z}$. The rotation mechanism is driven by a motor and an associated motion controller.
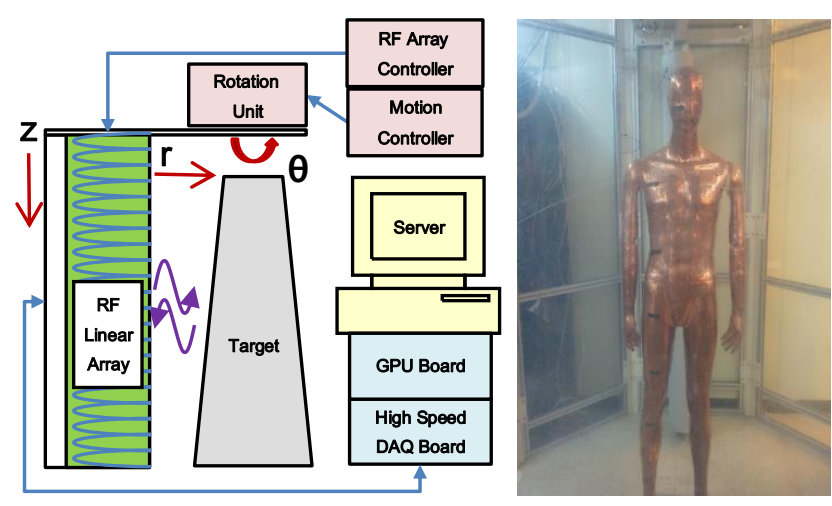

Fig.1. Schematic diagram for the scanning system and a photo of the target.

The antenna array moves around the body and acquires responses by comparing the transmitted and received (TX and RX) RF waves. Transceiver pairs are activated sequentially during scanning. Individual antennas in the array are sequentially switched on when the antenna controller receives the activation trigger signal. The differences in the RF wave between the TX and the RX is down-converted to analog signals that can be acquired by high-speed ADCs.

The motor and the antenna array are placed a couple of meters away from the server, hence, noise reduction must be considered in the communication and data lines. Two analog output channels are assigned for the I and Q signals that have conjugate pairs and symbolize the real and imaginary. The signals are then transferred with the trigger signals to the ADC. A significant number of IQ signals are stored in a large memory after sampling. The number of triggers is approximately $191 \mathrm{k}$ and the duration of the IQ signal is within 15 us. As significant quantities of data are generated from the high-speed sampling and frequency analysis, memory management is required. In the software structure, a large share memory (LSM) is defined and is accessible from the application software [16]. After scanning, the sampled signals in the time-domain are transformed to the frequency domain after frequency analysis.

\section{B. Frequency responses}

The IQ signals have sinusoidal patterns and contain information regarding the distance between the antenna array and the RF-reflective surface. The antenna array faces the target object in the $r$ (the cylindrical coordinate) direction, as shown in Fig.2. In this work, the antenna array is manufactured so that the peak frequency of the spectrum is inversely proportional to the distance. Therefore, the distance can be calculated by obtaining the maximum amplitude after the Fourier transform processing of the IQ signals as per following equation:

$$
F_{z \theta}(\omega)=\mathcal{F}\left[f_{z \theta}(t)\right]
$$

The FFT is useful in reducing the processing time [17] and the discrete Fourier transform is commonly used for finite sampled signals with a size $\mathrm{N}$, as follows: 


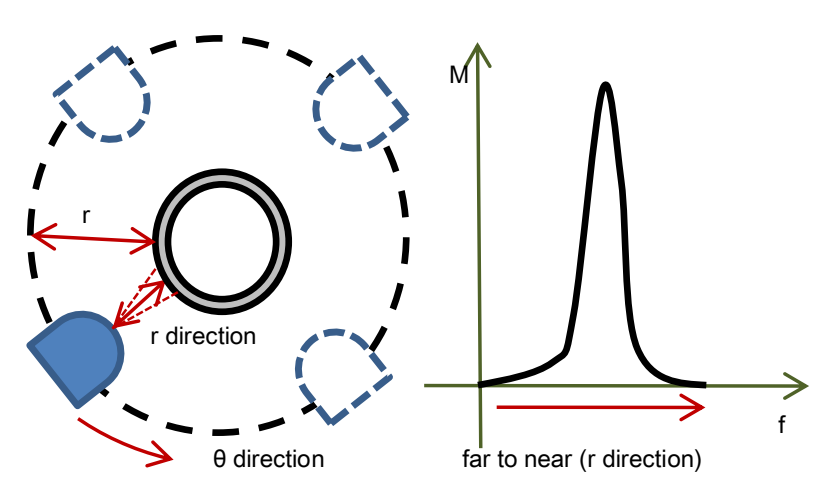

Fig.2. Relationship between the cylindrical coordinates and the frequency domain.

$$
F_{n, z \theta}(\omega)=\sum_{i=0}^{N-1} f\left(t_{k}\right) e^{-2 \pi k n / N}
$$

Then, the peak frequency at the maximum amplitude can be found by using optimum algorithms as well as by the equal step search method that is simple but effective [18]. This relationship can be written as follows:

$$
\max _{\omega_{\max }} F_{n, z \theta}\left(\omega_{k}\right) \quad(k=0 \sim N-1)
$$

Because individual antennas in the array are sequentially activated in the vertical direction, $\mathrm{z}$, this can be calculated using a trigger sequence. The array is rotated at a constant speed after acceleration. Therefore, $\theta$ can also be estimated with the trigger sequences; i.e., the scanning speed is almost constant and thus the $(\theta, \mathrm{z})$ position of the sampling signal can be estimated. Fig.3. shows the inverse linearity between the distance and the peak frequency. The distance can be calculated using a simple linear equation such as $a \omega_{\text {peak }}+b$. The position of the reflected surface $r_{z \theta}$ is obtained by inverse linearity and cylindrical coordinates, as follows:

$$
r_{z \theta}=r_{0}-\left(\mathrm{a} \omega_{p e a k}+b\right)
$$

\section{Zero-padding}

The resolution of the $r_{z} \theta$ is physically dependent on the wavelength of the millimeter wave but the distance can be estimated accurately using spectrum interpolation that considers floating point frequencies during the FFT. The FFT calculates the integer frequency from the sampled signal, and the precision of the spectrum can be increased by using a larger sampling size but this increases the measurement time owing to a large number of samples. The sampling size is consequently limited in the $3 \mathrm{D}$ RF measurement system, thus, spectrum interpolation of the floating point frequency can be considered for a higher distance accuracy. The sampling length is limited in typical $3 \mathrm{D}$ imaging systems and interpolation is applied to the frequency response using polynomial approaches [19].

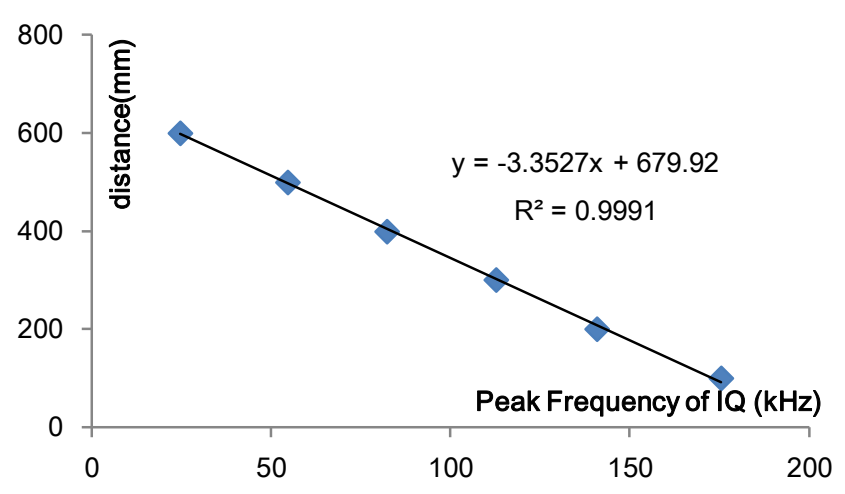

Fig.3. Inverse linearity between the peak frequency and the distance in the experiment.

Lagrange [20], smoothing [21], cubic spline [22], and window functions [23]. Zero-padding also provides spectrum interpolation and a floating point frequency for the time-limited sampling [24]. It is simple wherein, a zerofilled array is attached to the sampled signal. The signal after zero-padding has a size of $2^{m N}$, and FFT is applicable. Zero-padding is one of the most useful methods for detecting the peak frequency for smoothening RF images, and is used in magnetic resonance imaging (MRI) [25]. Thus, in this work, zero-padding is applied for 3D RF imaging and for obtaining the peak frequency accurately.

Current discrete Fourier transform ignores non-integer frequencies, but a spectrum interpolation can be achieved if the non-integer components are considered, that can be calculated by a division factor $\mathrm{m}$, as follows [26]:

$$
F_{n, z \theta}=\sum_{i=0}^{N-1} f\left(t_{k}\right) e^{-2 \pi k n / m N}(k=0 \sim m N-1)
$$

If $\mathrm{k} \geq \mathrm{N}$, there are no sampling data for $f\left(t_{k}\right)$, hence, they must be set to zero. $\mathrm{m}$ is generally a non-integer value and larger than $1.0 ; m=2^{M}$ for the purpose of applying computational codes and numerical algorithms. Zeropadding is attaching an $\mathrm{m}(\mathrm{N}-1)$ size zero array to the sampling signals. The sampling size acquired from the ADC has a limited value of $\mathrm{N}$ and the zero-padding array is extended to an $\mathrm{mN}$ size. If forward ZPFFT is applied to these zero-padding samples, the frequency responses will be interpolated. If inverse ZPFFT is applied to the interpolated spectrum, zero-padding samples from $1-\mathrm{N}$ will be reconstructed [27]. The following equation demonstrates that $1 / \mathrm{m}$ scaling of the frequency domain indicates $\mathrm{m}$ multiples of the sampling size and $\mathrm{m}$ divisions of the amplitude:

$$
\frac{1}{m} F_{z \theta}\left(\frac{\omega}{m}\right)=\mathcal{F}\left[f_{z \theta}(m t)\right]
$$

Zero-padding interpolates the spectrum below the decimal point, hence, we can estimate the peak frequency using a floating point number. The precision of the floating point usually shows $1 / \mathrm{m}$ in practical applications of distance measurements and the surface position can be detected more accurately. The 3D image by zero-padding appears smooth because of the interpolation effect. 


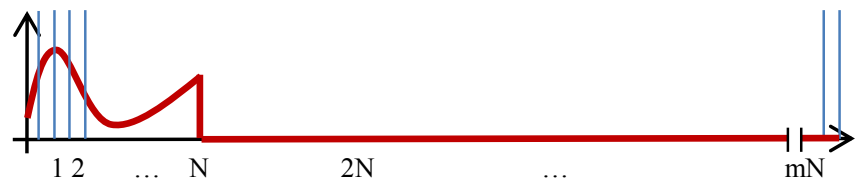

a)

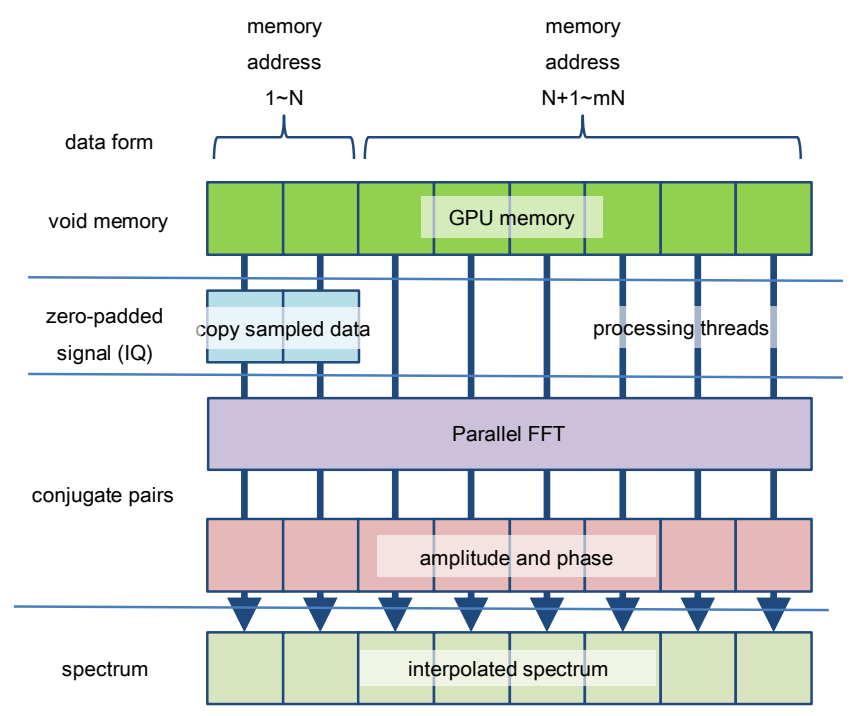

b)

Fig.4. Zero-padding concept a) Data allocation b) Parallel processing procedure of the ZPFFT.

\section{Parallel processing and spectrogram}

In this work, a GPU is applied to compute the ZPFFT because the sampling data is enormous; moreover, zeropadding expands the size of the sampling data and the FFT. The mathematical operations of the FFT can be accelerated using a GPU for RF signal analysis [28] as well as for zeropadding [29]. Zero-padding is implemented using a cuFFT that is a parallel version FFT, provided by the CUDA library. Owing to significant data transfer between the memory of the ADC and the GPU, the sampling data stored in the on-board memory of the ADC are sent to the main memory through direct memory access (DMA) transfer for the purpose of reducing the load in the central processing unit (CPU) and for reducing bottle necks in the data bus. Fig.4.a) shows that $(\mathrm{m}-1) \mathrm{N}$ zeros are attached to the $\mathrm{N}$ sized sampled signal IQ, before the FFT. Zero-padding can be achieved by copying the sampling data in the head of the void GPU memory with size $\mathrm{mN}$, as shown in Fig.4.b). Mathematical operations are performed on the individual GPU memory addresses using independent processing threads. Parallel FFT is conducted on the conjugate pairs of zero-padded data.

After the parallel FFT, the conjugate pairs are changed into amplitudes and phases. The GPU memory then contains the interpolated spectrum that is transferred to the PC memory. After all the zero-padding data are transformed to spectra, a set of spectra can be constructed for a crosssection in the $\mathrm{z}$ and $\theta$ directions.
Table 1. Specifications of the RF scanning system.

\begin{tabular}{cccc} 
Devices & Manufacturer & Shape/Model & Specification \\
\hline $\begin{array}{c}\text { Base } \\
\text { frame }\end{array}$ & - & $\begin{array}{c}\text { an octagonal } \\
\text { pillar }\end{array}$ & $\begin{array}{c}\Phi 2.2 \\
\mathrm{~h} 2.4 \mathrm{~m}\end{array}$ \\
Antenna & - & Array Type & $\mathrm{n}=191$ \\
& & & $\Delta=1 \mathrm{~cm}$ \\
ADC & Signaltec & PXI14400 & $\begin{array}{c}400 \mathrm{MHz} \\
14 \mathrm{bit}\end{array}$ \\
& & & $10.3 \mathrm{Tflops}$ \\
GPU & NVIDIA & C-2075 & $448 \mathrm{cores}$ \\
CPU & Intel & X5650 & $\begin{array}{c}2.66 \mathrm{GHz} \\
\text { hexa cores }\end{array}$ \\
OS & Microsoft & Windows7 & $64 \mathrm{bit}$ \\
Library & NVIDIA & CUDA & 4.0 \\
\hline
\end{tabular}

After the spectra are normalized, their amplitudes can be substituted to a color map, from blue to red, and the frequency axis can be placed in the $\mathrm{x}$ direction. If the spectra after color mapping are arranged in the $\mathrm{z}$ direction for a specified $\theta$, an image of the vertical cross-section can be constructed. If $\mathrm{z}$ is specified, the cylindrical coordinates are transferred into orthogonal coordinates using the following equation; from this the image, the horizontal cross-section can be obtained.

$$
(\mathrm{x}, \mathrm{y}, \mathrm{z})=(\mathrm{n} \cos \theta, \mathrm{n} \sin \theta, \mathrm{z}) \quad n=0 \sim n M-1
$$

The color-mapped images obtained on orthogonal coordinates are called spectrograms and are usually used in defect diagnosis and noise analysis [30,31]. (x, y, z) indicate the image coordinates based on pixels.

\section{EXPERIMENTAL PREPARATION}

\section{A. System}

A man-shaped dummy and a cylinder covered with copper foil are placed under the center of the rotational axis in the scanning system. An antenna array is rotated around the dummy and measurement is started after accelerating the rotation. The antenna array is considerably heavy and this causes a distortion of the rotation rod. Thus, a weight balance is attached to the opposite side of the array. A servo motor is connected to the center of the rotation rod during scanning. The servo motor is placed on the roof of a base frame in the shape of an octagonal pillar and made of aluminum. The antenna array is a board-band type and has a sweep range of $9 \mathrm{GHz}-18 \mathrm{GHz}$. A higher frequency band was used for RF imaging in previous studies, and the physical resolution of the image was better than that in our system. However, our system targets body measurements with clothing and penetration must be considered; therefore, our system is developed based on the X-Ku band. After the response of the RF wave is received using an antenna, it is 
down-converted to an analog signal about $20-180 \mathrm{kHz}$ and the analog signal is sampled using an ADC board. The ADC has a sampling rate of $400 \mathrm{MHz}$ and an on-board memory of $1 \mathrm{~GB}$. The raw data are sequentially stored in the on-board memory of the ADC during scanning and transferred to the shared memory between the triggers. The IQ signals, $f_{z \theta}$, are sampled using the 14-bit dual channels of a high-speed ADC during the rotation and transferred to the LSM through DMA. After scanning is completed, the data in the LSM are sent to a GPU through DMA by block transfer. The GPU performs a parallel FFT for the sampled signal after zeropadding is performed on the sampled signal. The number of IQ signals is $191 \mathrm{k}$ and the size of an individual sampling is $\mathrm{N}=2048$. The ADC, the GPU, and the $32 \mathrm{~GB}$ on-board memory are installed in a control PC. Multiple software applications interface the hardware devices that communicate with each other through a large shared memory and an inter-process communication (IPC). The result of the parallel ZPFFT is then returned to another LSM through DMA. The spectrograms are constructed for an operator specified $\mathrm{z}$ and $\theta$, and stored as bitmap files with pixel ranges of \pm 256 frequencies for the vertical crosssection and \pm 128 frequencies for the horizontal cross-section. Other frequencies are omitted because the amplitudes are considerably low and they appear blue only in the spectrograms. The applications are developed using Visual Studio 2008 and CUDA 4.0. Table 1. shows the specifications of the scanning system.

\section{B. Calibration}

The antenna array is composed of multiple antennas that have different amplitude and frequency responses in the spectrogram even if the same target is used. Fig.5. shows a spectrogram for a flat reflector in a stationary position before calibration when the distance is $400 \mathrm{~mm}$. The horizontal and vertical axes of the spectrogram indicate the distance and height, respectively. The color in the spectrogram varies from blue to red as the amplitude increases. Although the individual antennas are at the same distance from the reflector, individual spectra are discontinuous in the vertical direction. The figure shows that the intensities and the frequency responses of the spectra are different, implying that calibration must be done. In this study, intensity and distance calibrations are performed using an average function obtained from multiple spectrograms at different reflector positions. The spectrum of the i-th antenna, $F_{n, i}$, can be obtained at different distances and can be averaged by the following equation:

$$
G_{n, i}=\frac{1}{l} \sum_{j}^{l} F_{n, i}^{(j)}
$$

where, $\mathrm{G}$ is the average spectrum, 1 is the number of spectra, and $\omega$ is the frequency. The peak amplitude of the spectra can be found using the following equations:

$$
\begin{aligned}
& G_{\text {max }}=\max _{j} G_{j, i} \\
& F_{\text {max }}=\max _{j} F_{j, i}
\end{aligned}
$$

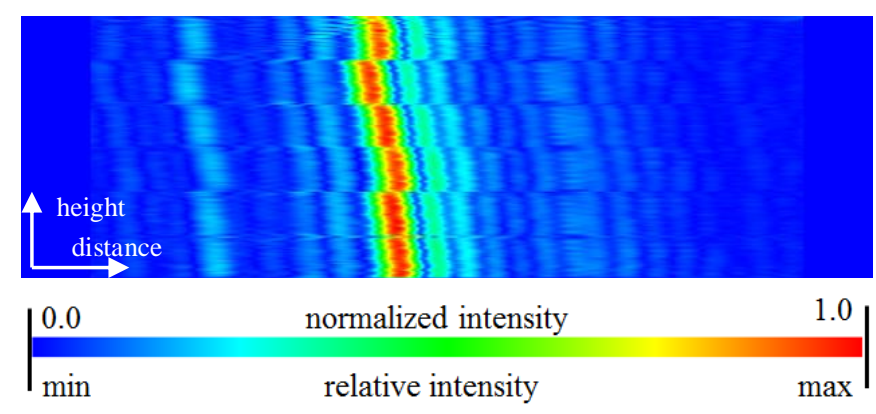

Fig.5. Spectrogram of a flat reflector before calibration, calculated by the ZPFFT.

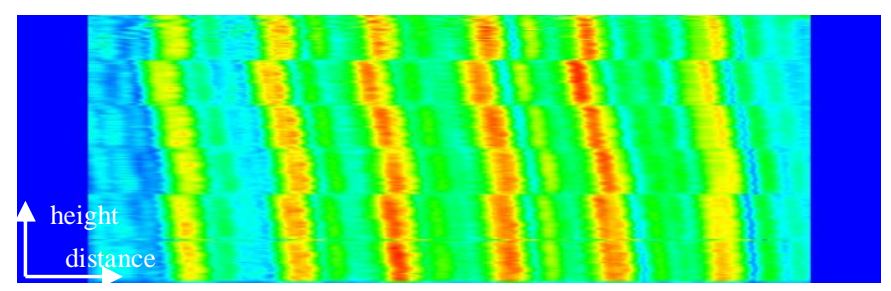

a)

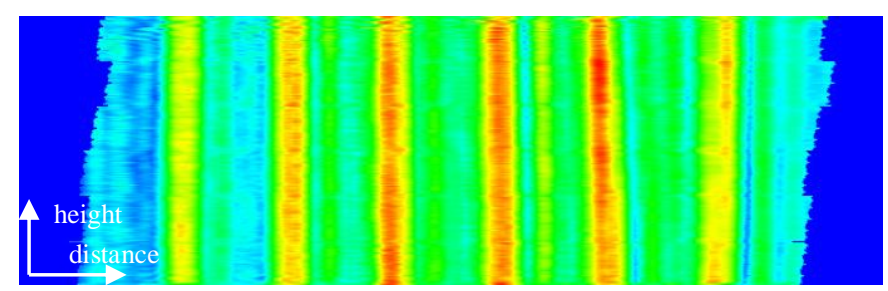

b)

$\left|\begin{array}{lcr}0.0 & \text { normalized intensity } & 1.0 \\ \min & \text { relative intensity } & \max \end{array}\right|$

Fig.6. superposed spectrogram of a flat reflector in different distances a) before calibration and b) after calibration; calculated by ZPFFT.

The amplitude can be calibrated by normalizing the spectra using the average spectrum given by

$$
F_{n, i}^{\prime}=\frac{G_{\max }}{F_{\max }} F_{n, i}
$$

where $F_{n, i}^{\prime}$, is the calibrated spectrum. The discontinuity can be removed by shifting the spectrum in the frequency direction. The amount of shift can be calculated by cross correlation. Normalized cross correlation, $\gamma$, is defined by, 


$$
\gamma_{i}=\frac{1}{N-1} \sum_{j}^{N} \frac{\left[F_{j, i}^{\prime}\left(\omega_{j}+\tau_{i}\right)-\bar{F}^{\prime}\right]\left[G_{j, i}-\bar{G}\right]}{\sigma_{F^{\prime} i} \sigma_{G}}
$$

where $\sigma$ is the standard deviation of the spectrum. The amount of the shift is determined at the point of maximum correlation when $\tau_{i}$ is varied within a certain range. Then, the distance calibrated image can be defined as follows:

$$
F_{n, i}^{\prime \prime}=F_{n, i}^{\prime}\left(\omega_{n}+\tau_{i}\right)
$$

Fig.6. shows a spectrogram obtained by superposing multiple spectrograms measured at different distances from the flat reflector. The red lines in the raw spectrogram are broken before calibration, as shown in Fig.6.a) and are formed after completing the amplitude and distance calibrations, as shown in Fig.6.b). As there is an inverse linearity between the peak frequency and the distance, the distance can be calibrated using a frequency calibration.

\section{RESULTS AND DISCUSSION}

A cylinder covered with copper-foil is scanned for verification of the final image using this complex signal processing. Fig.7.a) shows the RF image after applying FFT only, observed in a vertical plane. The two spectrograms are joined at $\theta=+180^{\circ}$ or do the spectrograms have a different quality at $\theta=+180^{\circ}$ ? The horizontal axis indicates the distance from the center, $r$, related to the frequency. The vertical axis represents the height, $z$, related to the antenna position in the array. A horizontal line in a fixed vertical position indicates a normalized spectrum after application of the calibration factors, thus, Fig.7.a) shows the spectra drawn in a rotational direction. The color in the spectrogram implies the normalized intensity of the normalized spectrum. The color changes from blue to red when the normalized intensity is varied from $0.0-1.0$. The high amplitude region is drawn in a non-blue color and located near the scanning center. The two red vertical lines in Fig.7.a) are clearly shaped, indicating the highest amplitude. This shape is the same as the cross-section of the cylinder in a vertical plane. Other lines of non-red colors are formed around the red line. A red line is also shown in Fig.7.b), similar to a circle, which is the cross-section of the cylinder in the horizontal plane. Circles of non-red colors are also formed around the red circle. Considering Fig.7.a) and Fif.7.b), the shapes of the red lines are equal to the shape of the cylinder. The shapes in non-red colors are caused by the echo signal that is inevitably detected in RF measurements.

Fig.8. shows the spectrogram obtained from the dummy man after applying the FFT only. The figures are constructed by joining two spectrograms at $180^{\circ}$, on the scanning center. Fig.8.a) illustrates the front view and the shape is similar to the outer profile of the dummy. In Fig.8.a), the head, arms, hands, and legs are approximately visible, but the chest, stomach, crotch, and the inner arms are not shown. This is caused by reflection surface in the RF direction. The RF signals emitted from the source are reflected to the outer arms and the RF signals perpendicular to the reflection surface are detected. Therefore, the RF signals could not reach the inner surfaces such as the chest and the crotch that do not appear in the spectrogram. Fig.8.b) shows the side view that depicts the head, chest, stomach, hips, and legs. The echo effect is also evident in the case of the dummy man. Fig.9.a) to Fig.9.d) show radial spectrograms in the horizontal planes with the measurement positions at the head, shoulder, hip, and legs. Radial spectrograms are constructed by mapping the normalized spectra with equal heights in the radial direction. A closed curve is constructed by connecting the non-blue points that have relatively higher amplitudes. The shapes of the closed curve in Fig.9. are similar to the body.

Table 2. Processing time for the computation methods.

\begin{tabular}{cccc}
\hline Methods & $\begin{array}{c}\text { Processing } \\
\text { time }\end{array}$ & $\begin{array}{c}\text { Math } \\
\text { operation } \\
\text { index }\end{array}$ & $\begin{array}{c}\text { Speed } \\
\text { index }\end{array}$ \\
\hline FFT (GPU) & $13 \mathrm{~s}$ & $22.5 \mathrm{k}$ & $5.8 \times 10^{-4}$ \\
FFT (CPU) & $95 \mathrm{~s}$ & $22.5 \mathrm{k}$ & $4.2 \times 10^{-3}$ \\
ZPFFT (GPU) & $43 \mathrm{~s}$ & $106.5 \mathrm{k}$ & $4.4 \times 10^{-4}$ \\
\hline
\end{tabular}

In this case, the inner arms, and inner legs are not depicted clearly. These inner surfaces are not easily exposed to antennas and are difficult for the RF wave to reach.

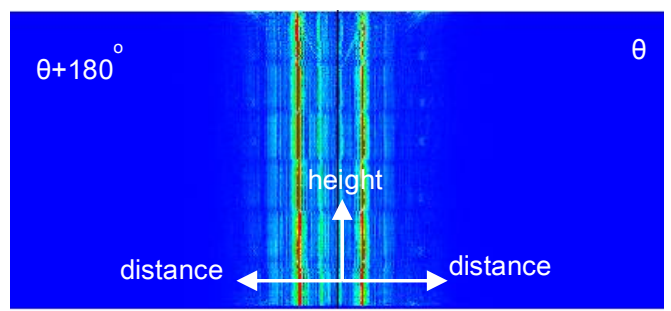

a)

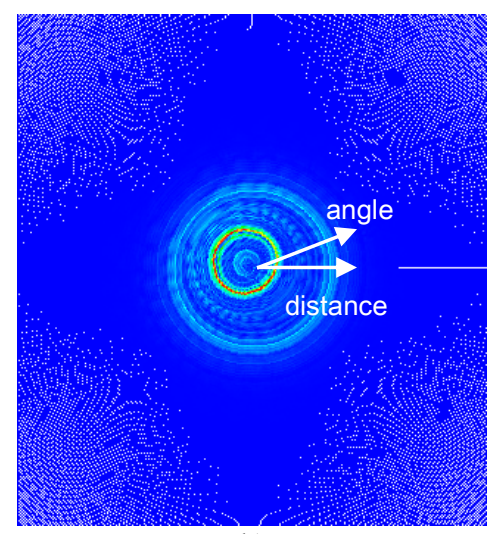

b)

$\left|\begin{array}{lcr}0.0 & \text { normalized intensity } & 1.0 \\ \min & \text { relative intensity } & \max \end{array}\right|$

Fig.7. Spectrogram after scanning a cylinder and applying the FFT only a) in a vertical plane b) in a horizontal plane. 
Fig.10. to Fig.12. display the spectrograms after application of the zero-padding, $\mathrm{m}=4$, using the raw data shown in Fig.7. to Fig.9. Fig.10. shows a spectrogram of the cylinder after applying zero-padding. A wall shape in the spectrogram of Fig.10.a) is formed and a circle is depicted in Fig.10.b). These shapes are shown more clearly than in Fig.7. after application of the zero-padding. This is because the high amplitude region of the vertical spectrogram after zero-padding is extended and the shape of the horizontal spectrogram is larger than that of Fig.7. The central width between the red lines in Fig.7. is 44 pixels, and that in Fig.10.a) is 172 pixels. The width after applying zero padding is approximately four times higher than that after the FFT only. However, echo patterns around the cylinder also increase after applying the zero-padding because the difference between the 1 st and the 2 nd peak had decreased.

Fig.11. shows the spectrograms of a dummy man after application of zero-padding. Fig.11.a) is the front view and Fig.11.b) shows the side view. The inner side of the arms and legs is not depicted in the figures. The spectrograms of Fig.11. are enlarged compared to those in Fig.8. but the echo signal also increases. Fig.12. displays the radial spectrograms measured in the same positions as in Fig.9. The shape also shows enlarged figures in the radial direction compared to Fig.9. A closed curve is formed by connecting the high amplitude points. Spectrograms measured for a dummy man in Fig.11. and Fig.12. exhibit an improvement in the image quality compared to those in Fig.8. and Fig.9.

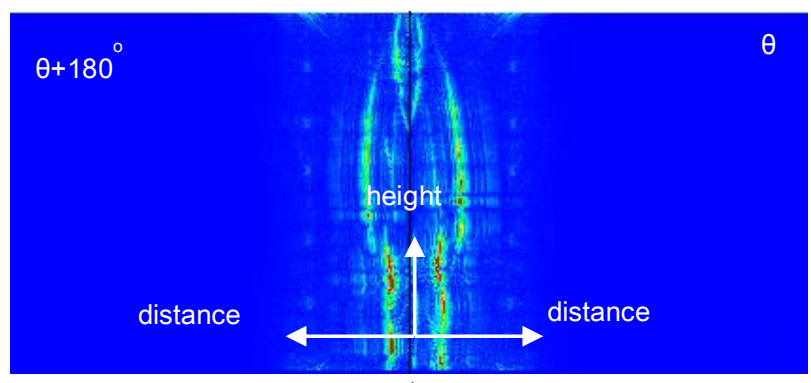

a)

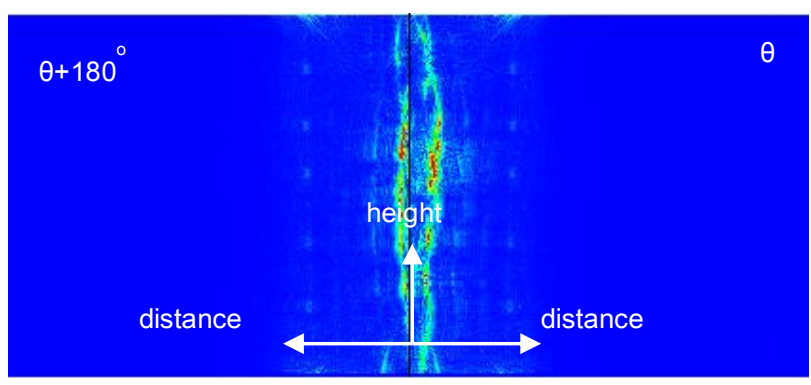

b)

$\left|\begin{array}{lcr}0.0 & \text { normalized intensity } & 1.0 \\ \min & \text { relative intensity } & \max \end{array}\right|$

Fig.8. Vertical cross-section synthesized from spectrograms after FFT computation only a) front view b) side view.

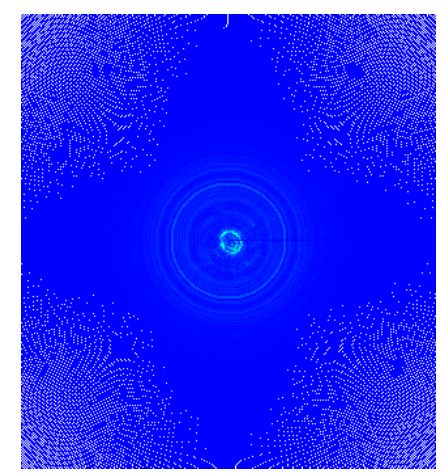

a)

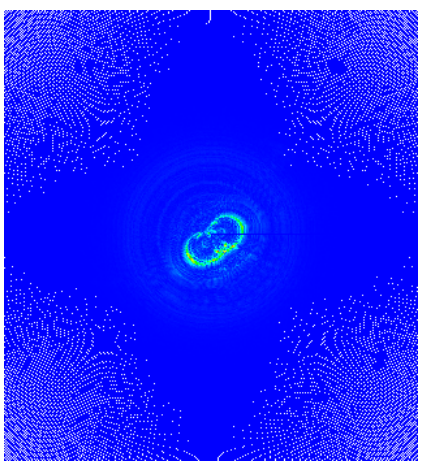

b)

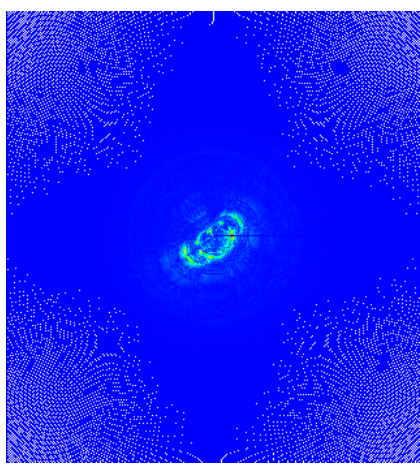

c)

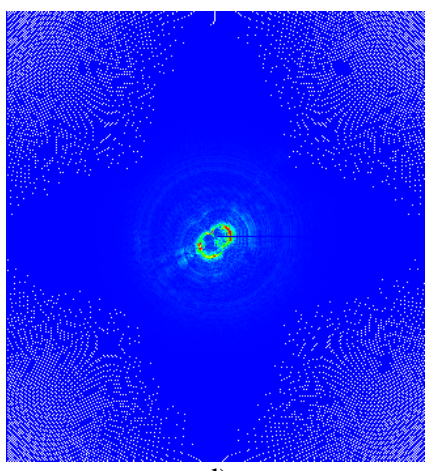

d)

$\left|\begin{array}{lcr}0.0 & \text { normalized intensity } & 1.0 \\ \min & \text { relative intensity } & \max \end{array}\right|$

Fig.9. Horizontal cross-sections synthesized from spectrograms after FFT computation only a) head b) shoulder c) hip d) legs. 


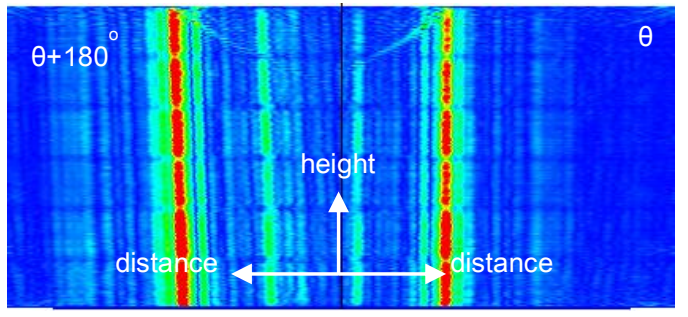

a)

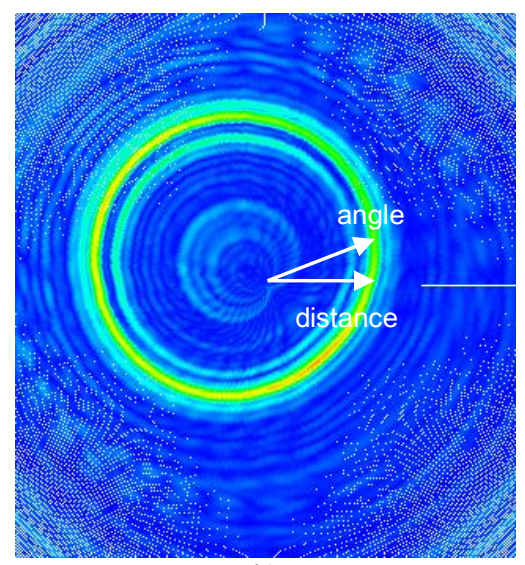

b)

0.0 normalized intensity relative intensity 1.0

$\min \quad$ relative intensity $\max$

Fig. 10. Spectrogram after scanning a cylinder and applying zeropadding FFT a) in the vertical plane b) in the horizontal plane.

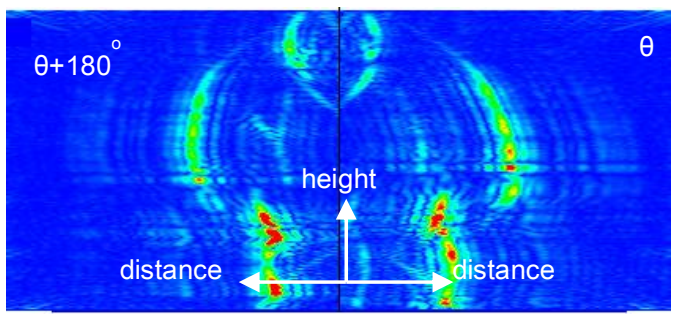

a)

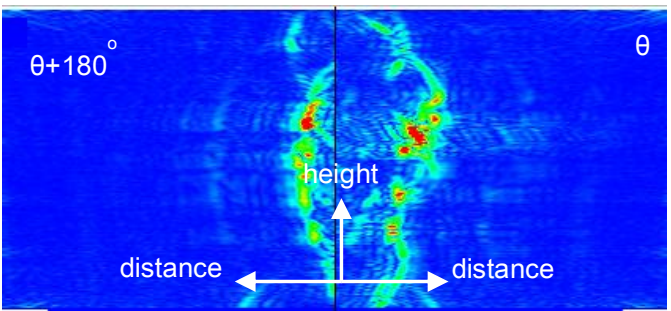

b)

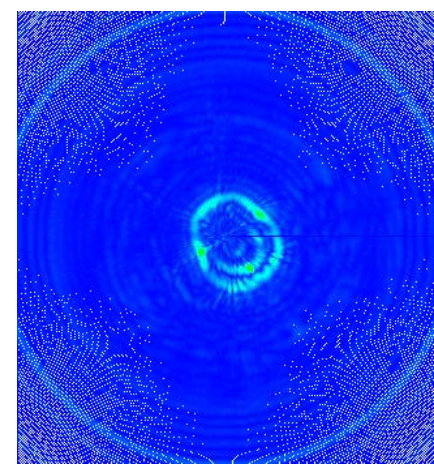

a)

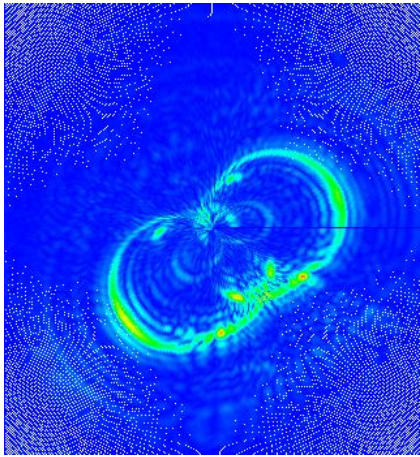

b)

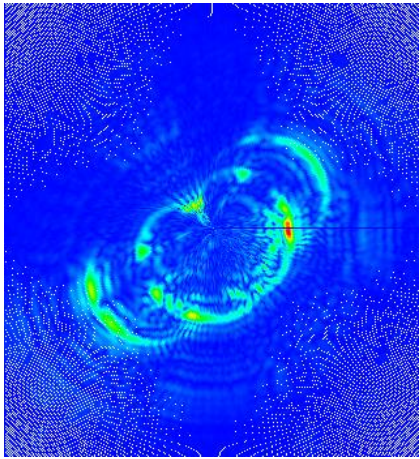

c)

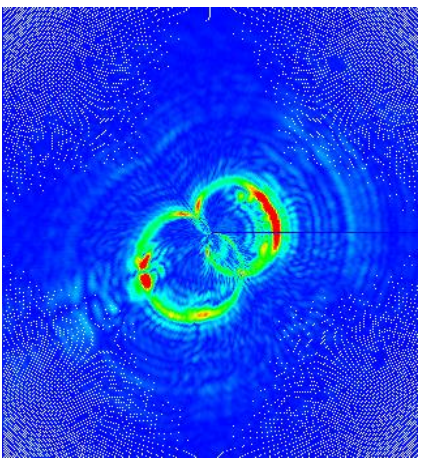

d)

$\left|\begin{array}{lcr}0.0 & \text { normalized intensity } & 1.0 \\ \min & \text { relative intensity } & \max \end{array}\right|$

Fig.11. Vertical cross-sections synthesized from spectrograms using zero-padding FFT a) front view b) side view.

$\left|\begin{array}{lcr}0.0 & \text { normalized intensity } & 1.0 \\ \min & \text { relative intensity } & \max \end{array}\right|$

Fig.12. Horizontal cross-sections synthesized from spectrograms using zero-padding FFT a) head b) chest c) hip and d) legs. 
Table 2. displays the processing times of the GPU-FFT, the CPU-FFT, and the GPU-ZPFFT for $191 \mathrm{k}$ points. The processing time is $13 \mathrm{~s}$ for the GPU-FFT, $95 \mathrm{~s}$ for the CPUFFT, and $43 \mathrm{~s}$ for the GPU-ZPFFT, respectively. The shortest time is for the GPU-FFT and the longest for the CPU-FFT. The number of mathematical operations can be estimated using $\mathrm{Nlog}_{2} \mathrm{~N}$ for the FFT and $\mathrm{mNlog}_{2} \mathrm{mN}$ for the zero-padding FFT. The speed index is obtained using the processing time and the mathematical operations. A low speed index indicates a high efficiency of computation and is lowest for the GPU-ZPFFT. The results indicate that zeropadding is beneficial for the RF scanning system and possibly increases the computational efficiency. As $\mathrm{m}$ increases, the high amplitude region of the spectrogram increases, thus, the distance accuracy can increase proportional to $\mathrm{m}$. However, the amplitude decreases to $1 / \mathrm{m}$ and the spectrum is distorted owing to the echo signal. Therefore, there will be a limit to the increase in $\mathrm{m}$. The limit was approximately $\mathrm{m}=32$, in the experiment.

The speed index can be defined by dividing the processing time by the math operations, indicating the processing efficiency. The index shows that the zero-padding FFT is more efficient than the other methods considering the number of mathematical operations. From these facts, it is demonstrated that the GPU is useful for detecting a 3D profile of the whole body for millimeter imaging. The data processing method proposed in this work clearly provides a higher distance accuracy and reduces the processing time for computing a GB size of data.

\section{CONCLUSION}

In this work, a body scanning system was adopted for detecting the 3D profile of a clothed man using a GPU and a ZPFFT. The scanning system was composed of an RF antenna array, a motion unit, controllers, a high-speed ADC and a GPU. The parallel processing unit, the GPU, was applied to a conventional cylindrical body scanner to accelerate the enormous amount of computations of the ZPFFT.

The hardware devices and software applications were designed considering the large quantities of data and computations. The body scanner acquired large signal data that was transformed into frequency-domain data using the ZPFFT. Because the antennas in the array had different characteristics, a calibration method was presented using an average function and a flat reflector. The results showed that the shape of the human dummies detected after scanning and zero-padding can provide a better distance accuracy for the spectrograms. The peak frequency in the interpolated spectra can be detected with a $1 / \mathrm{m}$ distance accuracy, spanning a non-integer frequency range. It is expected that the ZPFFT adopted in this work will not only be useful for achieving a higher distance accuracy but also for body index measurements in the apparel industry.

\section{ACKNOWLEDGMENT}

This work was funded and supported by the Korea Institute of Industrial Technology (http://www.kitech.re.kr). This research is one of the capability reinforcement projects branched from the authorized mission of the Institution. (PEO16340)

\section{REFERENCES}

[1] Mizuno, K., Wagatsuma, Y., Warashina, H., Sawaya, K., Sato, H., Miyanaga, S., Yamanaka, Y. (2007). Millimeter-wave imaging technologies and their applications. In IEEE International Vacuum Electronics Conference, 15-17 May 2007. IEEE, 1-2.

[2] Schiessl, A., Ahmed, S.S. (2009). W-band imaging of explosive substances. In European Radar Conference (EuRAD 2009), 30 Sept. - 2 Oct. 2009. IEEE, 617620.

[3] Bjarnason, J.E., Chan, T.L., Lee, A.W.M, Cells, M.A., Brown, E.R. (2004). Millimeter-wave, terahertz, and mid-infrared transmission through common clothing. Applied Physics Letters, 85 (4), 519.

[4] Sheen, D., McMakin, D., Hall, T.E. (1998). Cylindrical millimeter wave technique for concealed weapon detection. In Proceedings of SPIE, 3240, 242250.

[5] Dallinger, A., Schelkshorn, S., Detlefsen, J. (2005). Short distance related security millimeter-wave imaging systems. In German Microwave Conference (GeMiC 2005), 5-7 April 2005, 244-246.

[6] Martin, C.A., Kolinko, V.G. (2004). Concealed weapons detection with an improved passive millimeter wave imager. In Proceedings of SPIE, 5410, 252-259.

[7] McMakin, D.L., Keller, P.E., Sheen, D.M. Hall, T.E. (2009). Dual surface dielectric depth detector for holographic millimeter-wave security scanners. In Proceedings of SPIE, 7309, $73090 \mathrm{G}$.

[8] Sheen, D., McMakin, D., Barber, J., Hall, T.E., Severtsen, R.H. (2008). Active imaging at $350 \mathrm{GHz}$ for security applications. In Proceedings of SPIE, 6948, 69480M.

[9] Tan, W.X., Hong, W., Wang, Y.P., Wu, Y.R. (2010) A novel spherical-wave three-dimensional imaging algorithm for microwave cylindrical scanning geometries. Progress in Electromagnetics Research, 111, 43-70.

[10] Northeastern University. F2-G: Advanced imaging technology for whole body imaging. Annual Report.

[11] D'Apuzzo, N. (2009). Recent advances in 3D full body scanning with applications to fashion and apparel industry. In Optical 3-D Measurement Techniques IX. Vienna, Austria: Janetschek.

[12] McMakin, V. (2008). Millimeter wave technology meets the market. PNNL-SA-55316, Pacific Northwest National Laboratory. http://availabletechnologies. pnnl.gov/media/35_115200920658.pdf.

[13] McMakin, D.L. 3D holographic digital body measurements for health and fitness monitoring. Pacific Northwest National Laboratory, http:// availabletechnologies.pnnl.gov/media/35_1152009205 23.pdf.

[14] Belega, D., Petri, D., Dallet, D. (2016). Impact of harmonics on the interpolated DFT frequency estimator. Mechanical Systems and Signal Processing, 66-67, 349-360. 
[15] Kim, H.T., Kim, S.T., Jin, K.C. (2013). A parallel processing system for measuring 3D surface profile using RF spectrogram. In 23rd International Crimean Conference Microwave and Telecommunication Technology (CriMiCo), 8-14 September 2013. IEEE, 1060-1062.

[16] Kim, H.T., Jin, K.C. (2013). Multi-application and large shared memory in a mechatronic system for massive computation. Applied Mechanics and Materials, 307, 18-22.

[17] Press, W.H., Teukolsky, S.A., Vetterling, W.T., Flannery, B.P. (1992). Numerical Recipes in $C$ : The Art of Scientific Computing, 2nd ed. Cambridge University Press, 500-509.

[18] Arora, J.S. (1989). Introduction to Optimum Design. MacGraw-Hill, 291-295.

[19] Agcaoglu, O., Santhanam, B., Hayat, M. (2013). Improved spectrograms using the discrete fractional Fourier transform. In IEEE Digital Signal Procesing and Signal Procesing Education Meeting (DSP/SPE), 11-14 August 2013. IEEE, 80-85.

[20] Zeeshan, M., Khan, S.A. (2012). A modified FFT and biquadratic interpolation based algorithm for carrier frequency offset estimation in MC-CDMA based ad hoc networks. In International Conference on Emerging Technologies, 8-9 October 2012. IEEE, 1-6.

[21] Ma, W., Jia, Y., Xiao, G., Qiu, X. (2007). Study of energy spectrum smoothing and appraising smoothing goodness. In 8th International Conference on Electronic Measurement and Instruments (ICEMI '07). IEEE, 73-76.

[22] Hawkin, W.G. (1994). FFT interpolation for arbitrary factors: A comparison to cubic spline interpolation and linear interpolation. In IEEE Nuclear Science Symposium and Medical Imaging Conference, 30 Oct. - 5 Nov. 1994. IEEE, Vol. 3, 1433-1437.

[23] Wang, X., An, G., Ma, H. (2008). Correcting algorithm of DFT spectrum of pulsed radar signal based on cubic convolution interpolation. In Second International Symposium on Intelligent Information Technology Application, 20-22 December 2008. IEEE, 326-330.
[24] McKeon, R.T. (2006). Steganography using the Fourier transform and zero-padding aliasing properties. In IEEE International Conference on Electro/information Technology, 7-10 May, 2006. IEEE, 492-497.

[25] Ashikaga, H., Estner, H.L., Elliot, D.A.H., McVeigh, R., Halperin, H.R. (2014). Quantitative assessment of single-image super-resolution in myocardial scar imaging. IEEE Journal of Translational Engineering in Health and Medicine, 2 (1), 1-12.

[26] Kim, H.T., Jin, K.C., Kim, J.S. (2014). Parallel processing algorithm for a direct solution of customized Fourier transforms. In ASME International Mechanical Engineering Congress and Exposition, 1420 November 2014. ASME. Vol. 4B, 36577.

[27] Kim, H.T., Jin, K.C., Kim, S.T. (2014). Frequency analysis and interpolated spectra of time-limited samples in 3D RF imaging. In ASME International Mechanical Engineering Congress and Exposition, 1420 November 2014. ASME. Vol. 4B, 36576.

[28] Mundo, C., Adhinarayanan, V., Feng, W. (2013). Accelerating fast Fourier transform for wideband channelization. In IEEE International Conference on Communications (ICC), 9-13 June 2013. IEEE, 33693373.

[29] Vasilache, N., Johnson, J., Mathieu, M., Chintala, S., Piantino, S., LeCun, Y. (2015) Fast convolutional nets with fbfft: A GPU performance evaluation. In International Conference on Learning Representations ICLR (2015), 7-9 May 2015.

[30] Czarnecki, K. (2016). The instantaneous frequency rate spectrogram. Mechanical Systems and Signal Processing, 66-67, 361-373.

[31] Soetam, Y., Bito, Y. (2015). Detection of features of prosthetic cardiac valve sound by spectrogram analysis. Applied Acoustics, 89, 28-33.

Received October 28, 2015. Accepted March 31, 2016. 